\title{
50
}

\section{Open architecture environment for control engineering education}

\author{
Wojciech Grega \\ Technical University of Mining and Metallurgy \\ Krakow \\ Poland
}

David Doughty

Feedback Instruments Ltd

Crowborough

United Kingdom

\begin{abstract}
The integration of dynamic models from the various branches of control system engineering into laboratory settings is a familiar means of providing real experience to students. The main objective of this paper is to introduce the concept of open architecture application in educational control laboratories and to discuss the advantages of this approach. The presentation is based on the examples of open architecture demonstration tools developed in the Control Laboratory of the Institute of Automatics in Krakow. The application of these tools to a commercial digital servo trainer is also described. In contrast with other approaches the environment includes commercial analysis and simulation tools popular with control engineers.
\end{abstract}

Main conference themes: information technology, software

Educational areas: higher education

Study topics: science/engineering

Secondary keywords: control technology, experimental, modelling, open systems, programming, simulation 


\section{INTRODUCTION}

The laboratory teaching of control is an important way in which abstract concepts of control can be related to the design problems. Many of the students taking courses in system modelling, identification and control theory find it difficult to relate the theory to the physical world and become less motivated. Also, when the theory cannot be directly applied to industrial situations, control might be taught as an applied mathematics course.

The main objective in developing control technology demonstrators in the Control Laboratory of the Institute of Automatics in Krakow was to provide the students access to several bench scale physical systems for experimental verification of the fundamentals of control theory. From the beginning it was evident that the use of laboratory scale models which illustrate theory at work in practical situations, is beneficial in helping the students to understand analysis and design procedures and retain this knowledge (Fig. 1).
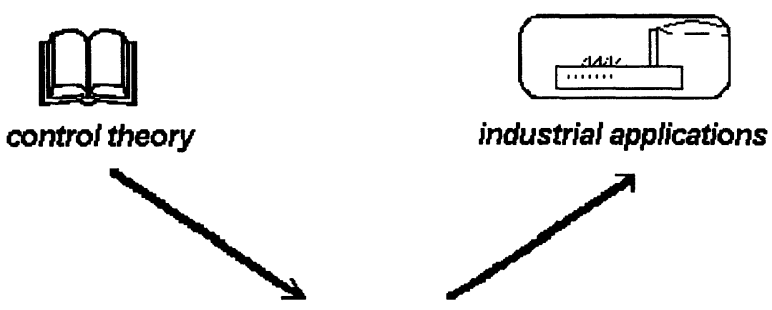

open-architecture demonstration tools

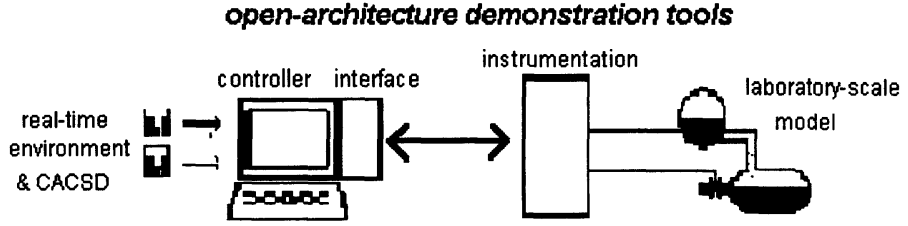

Fig. 1 Using laboratory scale models

Since the introduction of microprocessors new generations of digital controllers (PC compatible computers, programmable logic controllers (PLC) etc.) have been increasingly exploited for educational purposes. Digital controllers are plentiful, inexpensive and easily configured for changing requirements. They can easily interface with and control real-life processes.

The following representative areas were selected as the prototypes of realistic computer controlled processes from the various branches of control system engineering: electromechanical systems (inverted pendulum, helicopter, digital servo control), fluid-level control (multitank system), chemical process 
control (distillation column, PH-control). In most cases the technical importance of the models is immediately evident to the students.

The integration of these dynamic into laboratory settings is a familiar means of providing real experience to the students $[1,2]$. However, what makes our approach especially effective is the general assumption that the laboratory rigs are open systems. They use a standard hardware platform, a standard operating system and commercial Computer Aided Control Design Systems (CACDS) as engineering tools. Each laboratory station was built around a PC 386 computer equipped with a low-cost input/output interface. The real-time kernel (RTK) was developed under the MS-DOS operating system.

The laboratory programme is mainly based on projects during which the laboratory rigs are used for longer practical studies. A complete control engineering design starts from modelling and identification. After checking the validity of the developed models students have to simulate and analyze the dynamic properties of the system to be controlled. Finally, different control methods are to be applied and the performance has to be analyzed. Standard CACDS based on MathWorks [3] products provides software support for the projects. This environment consists of commercial MATLAB/SIMULINK packages and in-house developed specific toolboxes for laboratory rigs. The toolbox contains general purpose procedures for process communication, data visualization, etc., as well as specialized functions for process simulation and controller design. The basic functions of the toolbox are therefore in the user domain. Students may customize the toolbox to satisfy their requirements by extending, modifying or adding to it.

In experimental phase most students are surprised to find measured response deviating from simulated response. Justification for this requires an analysis of the physics of the process.

The main objective of this paper is to introduce the concept of open architecture applications in a control laboratory and to discuss some advantages of this approach. The presentation is based on the examples of open architecture demonstration tools developed in the Control Laboratory of the Institute of Automatics in Krakow. The application of a commercial laboratory rig is described in the last section.

\section{OPEN ARCHITECTURE COMPUTER SYSTEMS FOR CONTROL APPLICATIONS}

In the computer industry, the key term in recent years has been 'open system'. An open system follows industry standard interfaces so that hardware and software from different vendors can be integrated into a system without 
customized interfaces. This approach has been enormously effective on the personal computer market and appears to be so in the workstation market.

This experience is being repeated in the control industry. Open digital control systems by their nature enforce standard interfaces and encourage the use of standard hardware platforms, operating systems and CACDS tools. Most of the basic information on open systems is in the public domain making it easy to find out how these systems work. These trends offer new educational opportunities.

The open architecture process automation system (Table 1) combines process control functions (in a single or distributed system) with CACDS tools. It is a cost effective solution which allows execution on the same hardware platform of a growing list of advanced control algorithms, including intelligent control algorithms (fuzzy, expert etc.).

Table 1

\begin{tabular}{|l|l|l|}
\hline & Low-cost open system & Advanced open system \\
\hline hardware & industrial PC, VXI based PC, & $\begin{array}{l}\text { SUN/RISC workstation } \\
\text { (supervising control) }\end{array}$ \\
\hline interface: & $\begin{array}{l}\text { multifunction process interface } \\
\text { boards [5] }\end{array}$ & $\begin{array}{l}\text { IBM-PC (intelligent process } \\
\text { interface) }\end{array}$ \\
\hline operating system: & $\begin{array}{l}\text { real time kernel of DOS operating } \\
\text { system, or DOS+Windows for } \\
\text { decentralised configuration (Fig.2b) }\end{array}$ & $\begin{array}{l}\text { RTK in DOS environment for } \\
\text { process interface, UNIX for } \\
\text { workstation }\end{array}$ \\
\hline CACDS & $\begin{array}{l}\text { MATLAB/SIMULINK [3],[8] for } \\
\text { process analysis and controller } \\
\text { design, object oriented tools for } \\
\text { graphical interface development }\end{array}$ & $\begin{array}{l}\text { SIMULINK+C-code generator } \\
\text { for automatic development of } \\
\text { control algorithms. }\end{array}$ \\
\hline communication: & RS 232/485, & Ethernet \\
\hline
\end{tabular}

Figure 2 shows three typical configurations of open architecture control systems. Two major functions of the system can be identified: real-time data acquisition and control, and computer aided control system design/analysis which can be combined in one computer (Fig. 2a) or distributed (Fig. 2b., 2c).

Fig. 2a

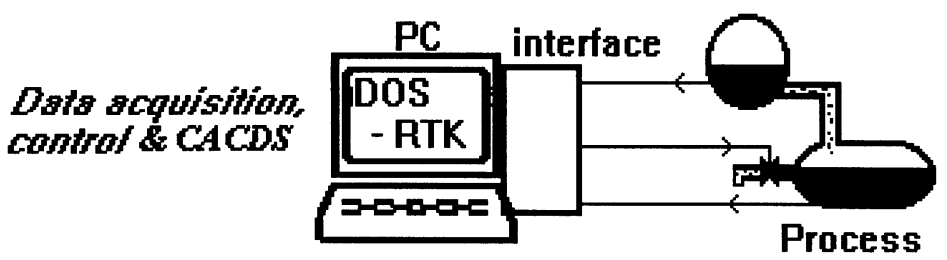


The solutions from figures $2 \mathrm{a}$ and $2 \mathrm{~b}$ are low cost and were selected for educational applications in the Control Laboratory.

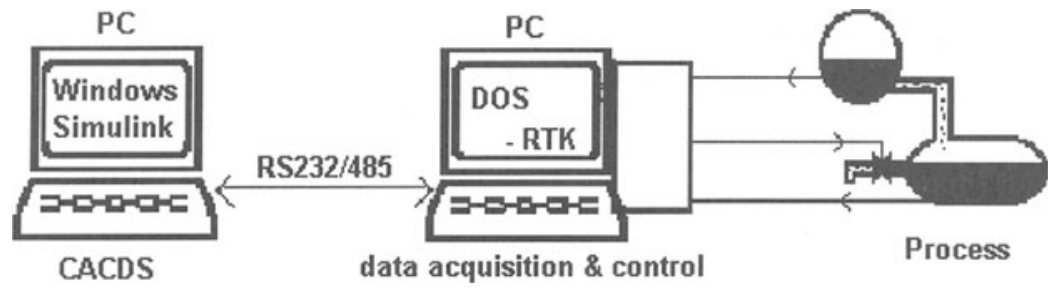

Fig. 2b

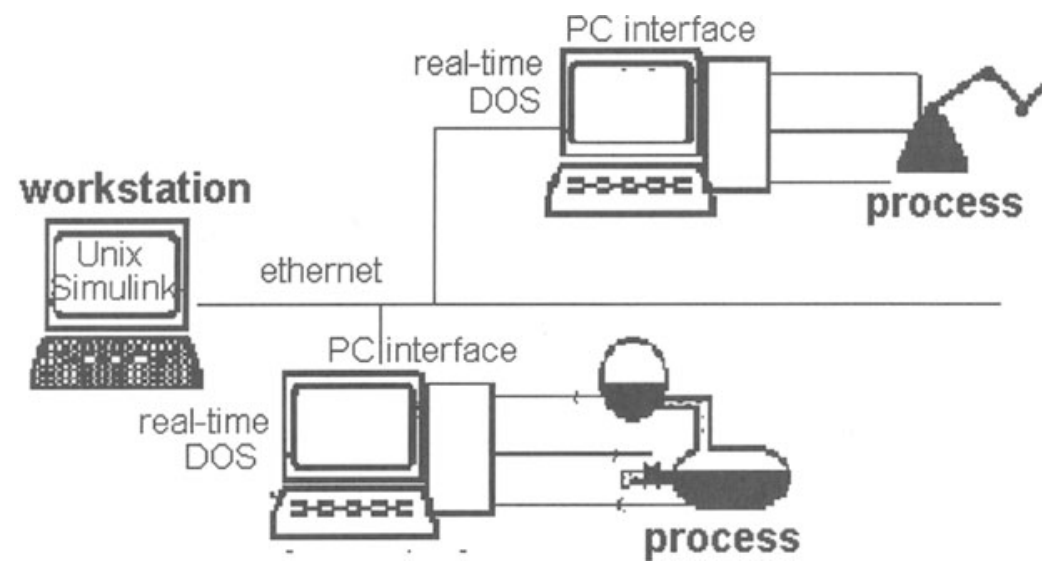

Fig. 2c

\section{LABORATORY DEMONSTRATION TOOLS}

The original motivation behind constructing laboratory scale models was to offer the students an environment for a complete engineering design, starting from modelling and simulation and ending with the experimental verification of a wide range of digital control strategies. The general idea was to capture the realism of industrial control problems in a laboratory environment. For this reason the development of the models in many cases was stimulated by industrial-commercial applications of new results.

The use of laboratory demonstration tools to teach control engineering was started ten years ago. The following models of dynamic systems were developed. 
Each laboratory station has three units of equipment (Fig. 1): laboratory scale model, instrumentation module, PC computer and interface as controller. The instrumentation module provides the appropriate converters, amplifiers and signal conditioning boards enabling transmission of the signals to the controller. The amplifiers provide the control signals to the actuators. The specialized signal conditioning boards are computer interfaces. These can be simple parallel digital input/output modules or multifunction data acquisition boards. which handle a variety of different input signals. The module contains a 12-bit A/D converter which offers a good representation of the original analogue signals. Input/output operations can be easily programmed in standard high level languages.

\section{Helicopter model}

The model consists of an arm pivoted at the centre with electrical motors mounted at each end. The motors drive two rotors: the main rotor and the tail rotor. Angular velocities of both rotors and the space position of the system arm are measured by tachogenerators and angle transducers. The model represents an interesting example of a nonlinear unstable multi-input, multi-output system (MIMO) of the third order with strong interactions.

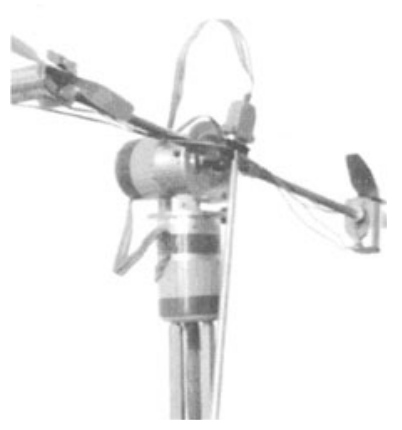

Fig. 3

\section{Inverted pendulum [4]}

The model consists of a pendulum hinged to a cart which moves along a rail driven by a DC motor. The position of the cart and angular position of the pendulum are sensed by Gray code transducers. The mathematical model consists of four nonlinear differential equations. The model is used to demonstrate the dynamics of an unstable system. The dynamic behaviour of the model relates closely to the type of problem encountered in the starting rocket problem.

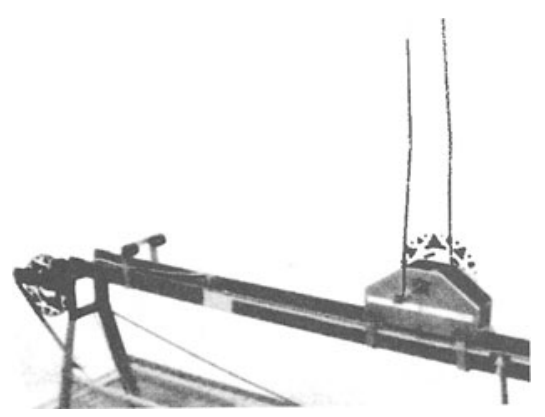

Fig. 4 


\section{Coupled-tanks system}

The model consists of an upper tank having constant cross section, and the lower tank of a cylindrical shape having variable cross section. Water is pumped into the first tank from the auxiliary compensatory tank by a pump driven by a DC motor. The water flows freely from the upper tank into the lower tank and finally out to the auxiliary tank. The limitations to the flows come from the orifices.

The size of the orifice can be varied by plugging and unplugging the holes with stops. The levels in the tanks are measured with pressure transducers. The general control strategy is to stabilize the level in the lower tank by adjustment of the pump operation. Although the dynamics of the fluid level systems are relatively straightforward, they involve challenging problems for tanks where the cross section varies with the fluid level. In this case the dynamics of the flow can

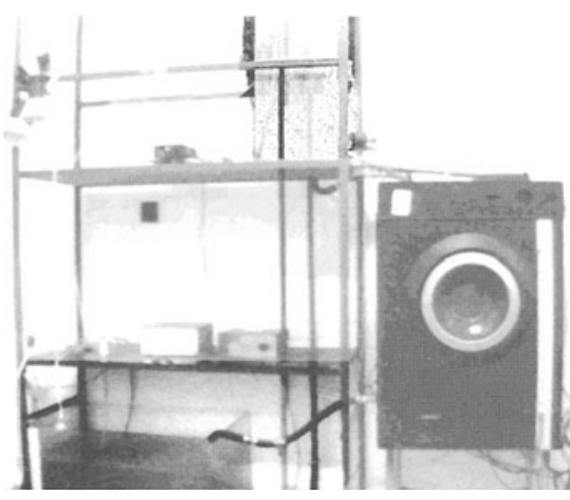
change extensively with the operating conditions of the process due to the nonlinearity between the level and the fluid volume in the tank.

\section{Digital Servo [10]}

The development of control technology demonstration tools in the Control Laboratory is carried out in cooperation with Feedback Instruments. This firm has gained experience in the production of training equipment for process control technology. One of the Feedback products is the 33-100 Servo Fundamentals Trainer [9], which demonstrates angular position and velocity control problems.

The 33-100 consists of a mechanical assembly and two separate control units

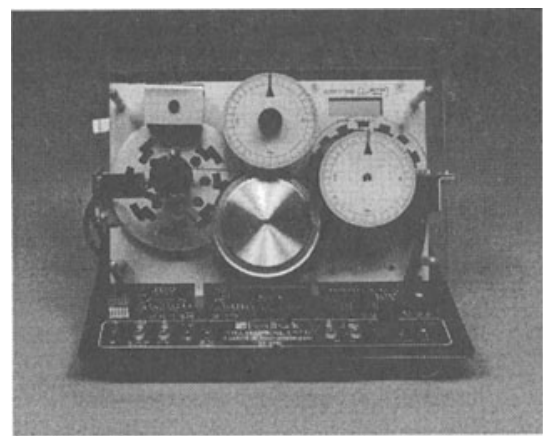
Fig. 6 covering analogue and digital control. The mechanical unit is used with either control unit as required and consists of a DC servo motor assembly with both 
analogue and digital position and velocity measurement sensors. A magnetic brake allows variations to be made to the load on the system. The digital unit provides $\mathrm{A} / \mathrm{D}, \mathrm{D} / \mathrm{A}$, signal conditioning and an excitation source. The configuration of the digital unit can be easily customized. The equipment is designed to investigate the principles of electro-mechanical control systems using a variety of analogue and digital control techniques. The application of the Servo Fundamentals Trainer for digital control is described in the last section of this paper as an example of an open architecture process automation solution.

A typical software structure under the DOS operating system for the laboratory station is illustrated in Fig. 7. It consists of a real-time kernel (RTK) and a computer aided control design system (CACDS). The real-time kernel has an interrupt controlled organization based on the internal MS-DOS clock working with a frequency of $18.2 \mathrm{~Hz}$. When the system controls a rapidly varying process the clock can be reprogrammed. The real-time kernel coordinates three basic tasks: process interfacing, data acquisition and control.

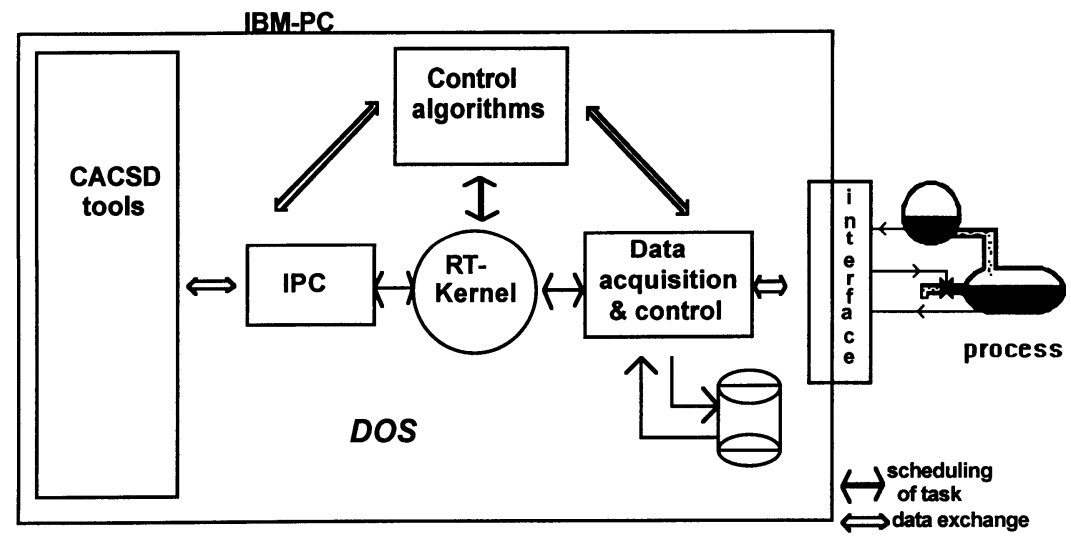

Fig. 7

The real-time applications are loaded as TSRs while the CACDS tools are running in the foreground. The IPC-unit (Inter-Process Communication) of the RTK provides via message queues. a communication service between the realtime application and the foreground CADS processes.

This configuration offers additional educational possibilities in real-time digital control. A student can write his own control interface while sampling periods are found via a laboratory experiment. The effects of sampling can clearly be demonstrated in this way. Also digital filtering algorithms can be 
included. Also other experiments can be carried out such as D/A and A/D conversion, signal transmission and sensor calibration.

\section{INTEGRATION OF COMPUTER AIDED CONTROL SYSTEM DESIGN (CACDS) AND REAL-TIME CONTROL}

A simplified development cycle of control system analysis and design is given in Fig. 8.

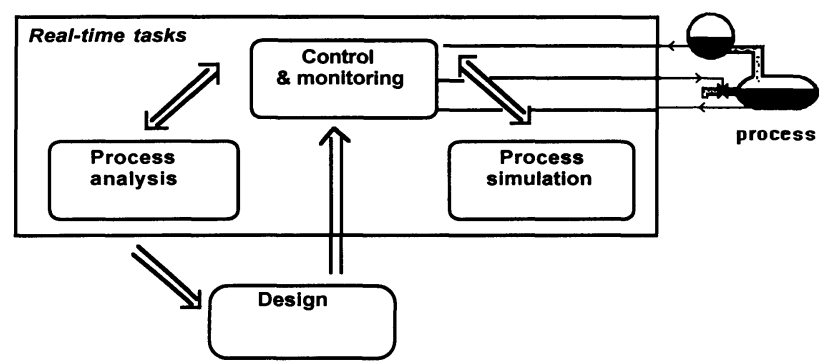

Fig. 8

Control and monitoring are procedures developed as tasks of the real-time system. Data collected by the data acquisition task can be analyzed in the online mode, using the identification toolbox. If necessary, the controller can be redesigned in off-line mode, verified by simulation and finally downloaded as code for the real-time controller. In many cases only modification of the controller parameters is necessary which can be obtained as output of the realtime simulation.

Educational activities in the Control Laboratory were intended to cover most of the cycle in Fig. 8. Clearly students then need control engineering "tools". The software environment developed is therefore an integrated environment, which supports two essential phases of control engineering:

- control system analysis and simulation;

- real time implementation of control algorithms.

In contrast with other approaches (A similar approach was recently reported at the Ruhr University Bochum [8]) the environment includes commercial analysis/simulation tools popular with control engineers. Powerful methods for designing digital control systems are based on Computer Aided Control Design Systems (CACDS). There is currently a number of professional commercial 
packages in use such as MATRIX $x_{x}[6]$, MATLAB [3] and ASCL [7] offering the control system engineer a complete set of tools for the simulation and analysis of automatic control systems. However, only a few CACDS are available to provide direct transition from the system design stage to its implementation.

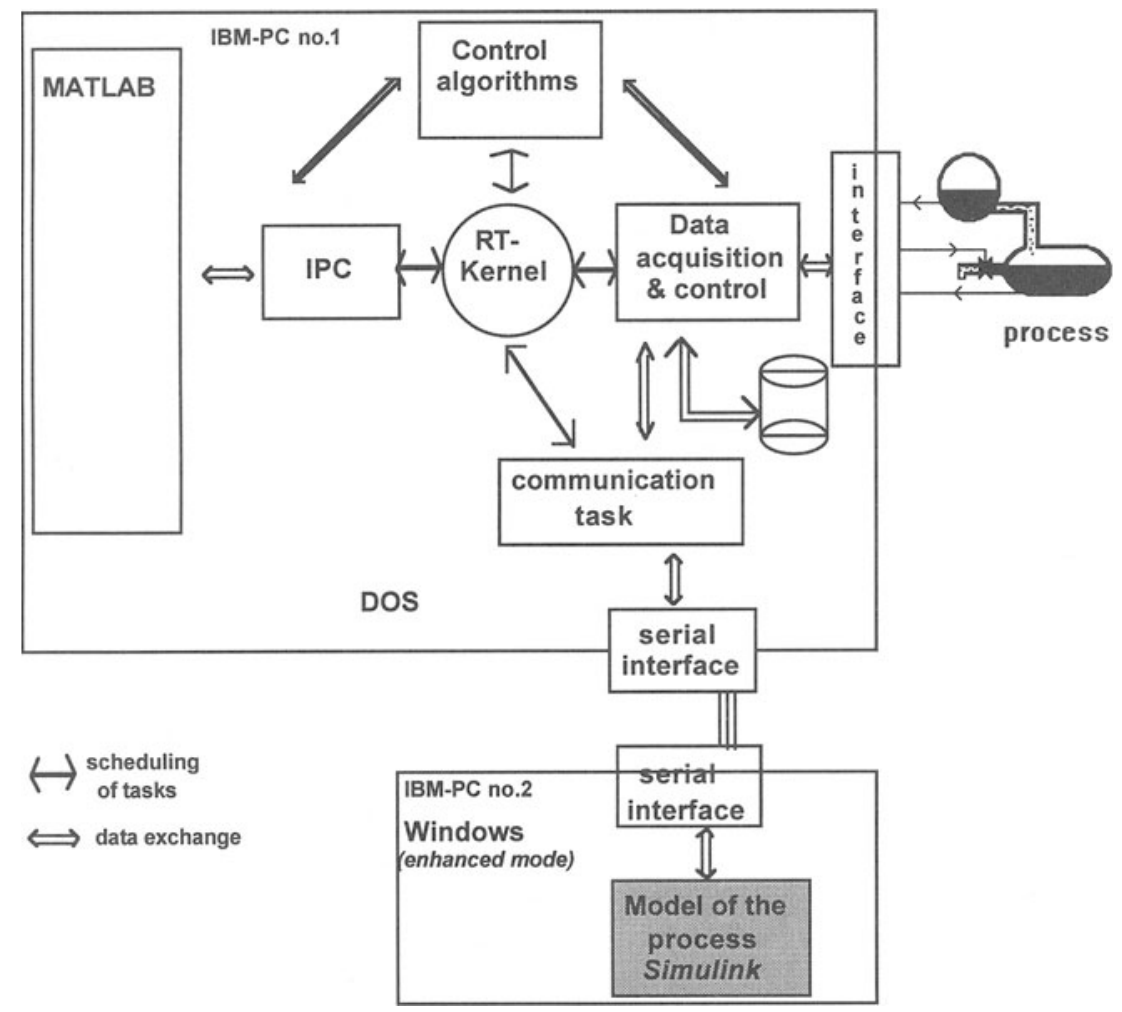

Fig. 9

MATLAB, a high-level modelling language popular among control engineers, is the CACDS tool for our laboratory open architecture environment. MATLAB offers toolboxes for signal processing, system identification and control design, both for linear and nonlinear problems, and SISO and MIMO systems. All of the above MATLAB facilities are available in the DOS environment, however process interface drivers are not included. To overcome this problem interprocess communication software modules were developed to allow data transfer between MATLAB and the real-time tasks (Fig. 7). 
The object oriented, dynamic system simulation package SIMULINK [9] was selected as an example of a modern simulation tool. SIMULINK must be started in the enhanced mode of MS-Windows operating system. In this mode Windows disables interrupts for fairly long time spans. Multitask operating systems cannot run in this environment. The integration of SIMULINK and the real-time control environment is therefore realized through a duplex (two computer) system consisting of two IBM-compatible computers (Fig. 2b).

Communication between the two PCs is achieved through RS-232 or RS485 serial links. Communication is synchronised through the internal clock and a suitable transmission protocol is specified. Message exchange is via this serial communications link (Fig .9).

\section{Example: Digital Servo Workshop}

The Digital Servo Workshop creates an open architecture environment for advanced control experiments using the Feedback ${ }^{\circledR}$ digital servo laboratory setup. The software approach applied in this case is illustrated in figure 10.

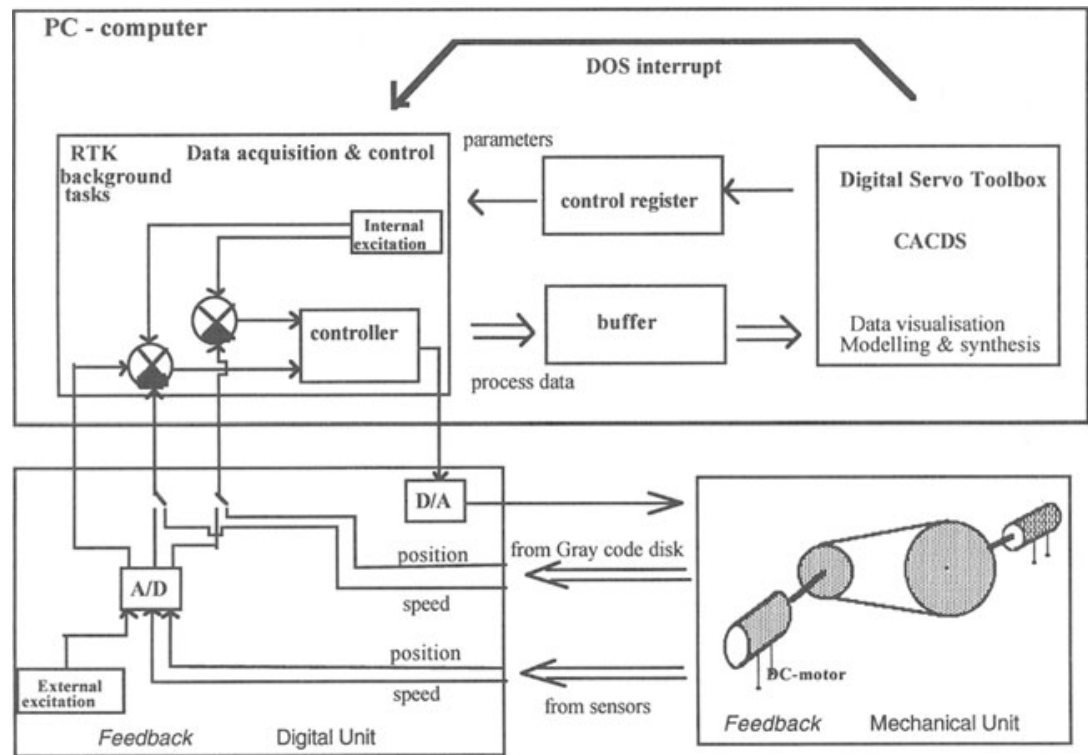

interface

laboratory-scale model

Fig. 10 
The Digital Servo Workshop consists of:

- Real-time kernel (RTK);

- Digital servo toolbox

The Real-time kernel runs in the background and supervises the real-time tasks shown in figure 11. These are $\mathrm{A} / \mathrm{D}, \mathrm{D} / \mathrm{A}$, digital input and digital output, control algorithms and tasks for communicating with the Digital Servo Toolbox. The DOS interrupt vector and register are to ensure the asynchronous information flow between the RTK and the Digital Servo Toolbox.

The Digital Servo Toolbox is a collection of M-files and C-code Mex-files which extends the MATLAB environment in order to solve digital servo modelling/design/control problems. The toolbox consists of three main sections: RTK communication, visualisation and simulation functions.

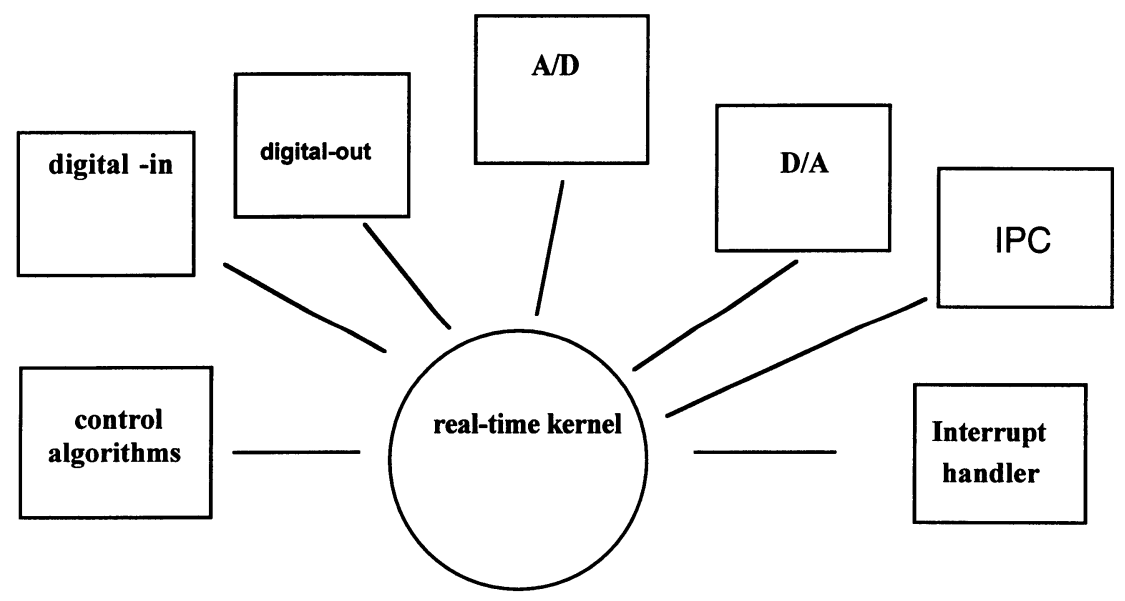

IPC - interprocess communication

Fig. 11

The RTK communication functions allow the configuration of real time tasks, set the control algorithm parameters and transmit data from the process to CACDS.

The visualization functions allow the user to customize visual windows, to plot process input/output data on the screen, etc. The simulation functions support the off-line design phase of control algorithm development. System 
behaviour can be simulated in open or closed loop modes with the application of different control algorithms such as PID, LQ, nonlinear (time optimal) and self tuning. The parameters calculated during the design phase can be directly transmitted to the real-time controllers.

The toolbox is an open system which inherently ensures that the basic functions of the toolbox are in the user domain. The functions described use the same standard as in the MATLAB environment. Users familiar with that environment can easily maintain their own systems, add new control algorithms or further customize these. A simple menu system is provided to simplify typical 'paths' through the toolbox, and to automate typical operations. This menu system contains a 'shell' function.

This environment allows use of advanced modern control techniques and is therefore suitable for both teaching and research purposes.

\section{CONCLUSIONS}

The laboratory experiments described support lectures in three major areas: control theory, modelling and real-time control. Students will find mathematical models used in the laboratory also in lectures and have a good background in CACDS applications. All of the courses associated with these laboratories have been well received by students.

It is our belief that an open architecture environment is the future for control engineering education. The development of CACDS tools which has been very rapid in recent years, will create new possibilities for education.

\section{REFERENCES}

1. Wellstead, P.E. (1990) Teaching control with laboratory scale models. IEEE Transactions on Education 33 (3) p. 286.

2. Grega, W and Maciejczyk, A. (1994) Digital Control of a Tank System. IEEE Transactions on Education 37 (8).

3. MATLAB Reference Guide, The Mathworks Inc., 1992.

4. Grega, W and Turnau, A. (1993) Laboratory models in control education. Proceedings of the 4th EAEEIE Conference: Multimedia and Computer Tools, Prague, pp. 134 - 139. 
5. MetraByte Corporation (1992) Data acquisition \& control boards, Product Selection Guide, Taunton, U.K.

6. Integrated Systems Inc. (1984) MATRIXX User's Guide. Santa Clara.

7. Mitchell and Gauthier Associates (1987) ASCL Reference Manual, edition 4.

8. Schmid Chr. (1992) Real-Time Control with CACDS-PC. Recent Advances in Computer-Aided Control Systems Engineering (ed M.Jamshidi), Elsevier, pp. 337-355.

9. The Mathworks Inc. SIMULINK-Release Notes,Version 1.2, May 1992.

10. Feedback Instruments Ltd. (1994) 33-100 Servo Fundamentals Trainer. 